\title{
Elevated Interleukin-6 Levels within 72 Hours Post Admission Are Associated with Disease Progression in Nonseptic Critically Ill Children
}

\author{
Lingfang Tan, ${ }^{1}$ Jianzhong Dang, ${ }^{2}$ Zhongping Liu, ${ }^{3}$ and Fang Zheng $\mathbb{D}^{1}$ \\ ${ }^{1}$ Department of Pediatrics, Union Hospital, Tongji Medical College, Huazhong University of Science and Technology, \\ Wuhan 430022, China \\ ${ }^{2}$ Department of Geriatrics, Renmin Hospital of Wuhan University, Wuhan 430060, China \\ ${ }^{3}$ Department of Hematology, Union Hospital, Tongji Medical College, Huazhong University of Science and Technology, \\ Wuhan 430022, China
}

Correspondence should be addressed to Fang Zheng; fangzheng99@sina.cn

Received 22 February 2020; Revised 28 May 2020; Accepted 19 June 2020; Published 10 July 2020

Academic Editor: Marija Mostarica-Stojković

Copyright (c) 2020 Lingfang Tan et al. This is an open access article distributed under the Creative Commons Attribution License, which permits unrestricted use, distribution, and reproduction in any medium, provided the original work is properly cited.

\begin{abstract}
It has been established that the innate and adaptive immune suppression was heterogeneous in septic and nonseptic critically ill patients, while the value of immune function in pediatric patients with nonseptic critical illness is limited. We conducted a single-center retrospective study to explore this issue. A total of 65 children with nonseptic illnesses were studied for lymphocyte subpopulations, immunoglobulin concentrations, complement concentrations, and cytokines in peripheral blood in the next 72 hours after admission to our Pediatric Intensive Care Unit (PICU). When compared to clinically recovered patients, patients with disease progression had a numerically lower but not significantly different median pediatric critical illness score and longer PICU median stays. The analysis of serum immunoglobulin (IgG, IgM, and IgA), serum complement (C3, C4) concentrations, and lymphocyte subpopulations showed no significantly difference between patients with and without relieved clinical symptoms by day 4 . For the cytokine analysis, the level of IL-6 was significantly higher in patients with disease progression than that in patients who clinically recovered $(p=0.046)$. In the univariate Cox regression analysis, plasma IL-6 levels were associated with outcome. Multivariate analysis evidenced that the level of plasma IL- 6 was one of the factors determining the length of hospital stays. In conclusions, our results demonstrate that increased IL-6 levels in the initial 72 hours post admission are associated with prolonged stays and disease progression in nonseptic critically ill children in the PICU.
\end{abstract}

\section{Introduction}

The immune system plays an important role in the acute phase of critical illness, as well as in late stage disease progression. Critical illness-induced immune suppression has been demonstrated in children with a variety of diagnoses, including sepsis [1], trauma [2], and cardiopulmonary bypass [3]. The most remarkable achievements of evaluating immune function of critical illness have been done in both children and adults in the field of sepsis. It has been established that blood levels of IL-6 had a diagnostic value and could predict the treatment success in sepsis [4-9]. The results from many studies showed that the innate and adaptive immune sup- pression was heterogeneous in septic and nonseptic critically ill patients $[10,11]$. In fact, acute bronchopneumonia was the most common disease in the PICU and the main causes of death included severe acute bronchial pneumonia, severe sepsis, complex congenital heart disease, severe cerebral trauma, respiratory failure, severe hand-foot-mouth disease, acute poisoning, and circulatory failure in China [12]. It suggested that the majority of critically ill children suffered from nonseptic disease in the PICU. However, the relationship between immune function and the prognosis of nonseptic critical illness in pediatric patients is poorly known yet.

We therefore investigated the early immunological characteristics in nonseptic critically ill pediatric patients. For 
this, immunomonitoring was performed during the three days after admission, which included lymphocyte subset count, plasma levels of immunoglobulin, and plasma cytokine concentrations: interleukin- (IL-) 2, IL-4, IL-6, IL-10, IL-17A, granulocyte colony-stimulating factor (G-CSF), granulocyte-macrophage colony-stimulating factor (GMCSF), tumor necrosis factor- (TNF-) $\alpha$, TNF- $\beta$, and IFN- $\gamma$. We anticipate that these data will support the value of early immune function evaluation in nonseptic children.

\section{Materials and Methods}

2.1. Patients. We retrospectively collected the information of pediatric patients ( $<14$ years old) who admitted between July 2018 and July 2020 to our PICU. The patients were identified from a computerized database at Union Hospital, Tongji Medical College, Huazhong University of Science and Technology. This study was approved by institutional ethics board of our medical college (No. 20200256). Patients with diagnosis of sepsis, immunodeficiency, or undergoing immunosuppressive therapy were excluded. Demographic information such as age and gender, the nature of underlying disease, and clinical status at admission to the PICU was abstracted on a standardized form. Details of the clinical manifestations and investigations including blood tests, blood gas analysis, liver and renal function tests, coagulation tests, genomics, and etiology tests were noted.

Pediatric critical illness score (PCIS) was evaluated by two attending physicians independently, and the results were averaged. Several parameters (including systolic and diastolic blood pressures, heart rate, respiratory rate, $\mathrm{PaO}_{2}, \mathrm{C}$-reactive protein (CRP), serum potassium, serum sodium, renal function, and gastrointestinal function) were collected at $72 \mathrm{~h}$ of PICU admission. The total possible score was 100 points, and the severity of disease was ranked as follows: $>80$ points, non-critical; $71-80$ points, critical; and $\leq 70$ points, extremely critical [13]. If PCIS points were more than 80 , the severity of illness was defined by the pediatric chronic complex condition [14].

2.2. Flow Cytometry. The blood samples were collected into tubes containing ethylenediaminetetraacetic acid from each patient in the first 72 hours after admission to the PICU. The evaluation of peripheral blood lymphocyte subpopulations including mature human $\mathrm{T}$ cells $\left(\mathrm{CD}^{+}\right), \mathrm{B}$ cells $\left(\mathrm{CD} 19^{+}\right)$, helper/inducer $\mathrm{T}$ cells $\left(\mathrm{CD} 3^{+} \mathrm{CD} 4^{+}\right)$, suppressor/cytotoxic $\mathrm{T}$ cells $\left(\mathrm{CD}^{+} \mathrm{CD}^{+}\right)$, and natural killer lymphocytes $\left(\mathrm{NK}, \mathrm{CD}^{-} \mathrm{CD}^{-} 6^{+} \mathrm{CD} 56^{+}\right.$) was performed by using flow cytometry (FACSCalibur, BD Biosciences, San Jose, California). The monoclonal antibodies used for the analysis of lymphocyte subsets were conjugated with fluorescein isothiocyanate (anti-CD3), phycoerythrin (anti-CD8, anti-CD56, and anti-CD16), and allophycocyanin (anti-CD19, antiCD4). All monoclonal antibodies were obtained from Becton-Dickinson (San Jose, CA).

In addition, plasma was obtained after centrifugation at $1,000 \times \mathrm{g}$ at $20^{\circ} \mathrm{C}$ for $20 \mathrm{~min}$ and immediately stored at $-80^{\circ} \mathrm{C}$ for the quantification of following cytokines: IL-2, IL-4, IL-6, IL-10, IL-17A, G-CSF, GM-CSF, TNF- $\alpha$, TNF- $\beta$, and IFN- $\gamma$, which were measured AimPlex bead-based immunoassays (AimPlex Biosciences, Inc., Pomona, CA).

2.3. Detection of Immunoglobulins. Serum immunoglobulin (IgG, IgM, and IgA) concentrations were measured by radial immunodiffusion enzyme assay using an automatic biochemical analyzer (Beckman, USA). Serum complement (C3 and C4) concentrations were measured by enzymelinked immunosorbent assay. All procedures were performed according to the manufacturer's instructions. All of measurement kits were purchased from Shanghai Kehua Biotechnology Co. Ltd.

2.4. Data Analysis. Quantitative parameters were described as the median (interquartile range (IQR)). All of these measurements were expressed as the mean \pm standard error (SE). Data were analyzed using GraphPad Prism 6.0 software (USA). Student's $t$-tests were used to compare continuous variables, and Mann-Whitney $U$ or Fisher's exact tests were used to compare categorical variables. The variables associated with a $p$ value $<0.10$ were included in logistic regression analysis. The $p$ values were two-tailed, and a $p$ value of less than 0.05 was considered statistically significant.

\section{Results}

3.1. Patients' Characteristics. During the period of July 2018 to July 2019, a total of 345 pediatric patients were admitted to our PICU, 286 patients fulfilled the inclusion criteria above specified, and immune function including evaluation of lymphocyte subset count, plasma levels of immunoglobulins, and plasma cytokine concentrations was performed in 65 patients. Demographic data are presented in Table 1. The main reasons for admission to the PICU included respiratory disease $(n=23)$, gastrointestinal disease $(n=15)$, and cardiovascular disease $(n=13)$. The median PCIS score was 85 , and the median PICU stay was 5 days.

3.2. Early Immune Function in Patients with Disease Progression versus Who Clinically Recovered. Baseline characteristics were comparable in patients who are recovered from clinical symptoms by day 4 and those who had disease progression (Table 1). When compared to clinically recovered patients, patients with disease progression had a numerically lower but not significantly different median (IQR) PCIS score [80 $(76 ; 90)$ vs. $86(80 ; 94)]$. What is more, patients with disease progression had a significantly longer PICU median stay [9 days (3-12) days] than that in patients with recovery [5 days (3-7) days].

Among patients with disease progression, serum immunoglobulin (IgG, IgM, and IgA) and serum complement (C3, C4) concentrations were similar to those found in clinically recovered patients. The analysis of lymphocyte subsets showed that patients with disease progression had lower but not significantly different level of B cells [22.26\% (13.72\%$26.52 \%)$ vs. $26.98 \%(19.83 \%-34.16 \%)]$ than that in clinically recovered patients. Meanwhile, patients with disease progression had higher but not significantly different median (IQR) levels of $\mathrm{CD}^{+} \mathrm{T}$ cells and $\mathrm{CD}^{+} \mathrm{T}$ cells than patients with recovery, respectively $[70.21 \%(61.92 \% ; 77.22 \%)$ vs. $63.06 \%$ 
TABLE 1: Clinical characteristics of nonseptic critically ill children in PICU with or without clinical recovery by day 4.

\begin{tabular}{|c|c|c|c|}
\hline \multirow{2}{*}{ Characteristics } & \multicolumn{2}{|c|}{ Clinical symptoms relieved at day 4} & \multirow{2}{*}{$p$ value } \\
\hline & Yes $(n=42)$ & No $(n=23)$ & \\
\hline Age (years), median (IQR) & $3.1(0.4 \sim 8.1)$ & $3.6(0.4 \sim 8.3)$ & 0.8472 \\
\hline Male/female & $27 / 15$ & $17 / 6$ & 0.93 \\
\hline \multicolumn{4}{|l|}{ Diagnostic at PICU admission } \\
\hline Respiratory & $15 / 42(35.7)$ & $8 / 23(34.8)$ & 0.9472 \\
\hline Gastrointestinal & $10 / 42(23.8)$ & $5 / 23(21.7)$ & 0.8583 \\
\hline Cardiovascular & $9 / 42(21.4)$ & $4 / 23(17.3)$ & 0.7068 \\
\hline Neurological & $6 / 42(14.3)$ & $4 / 23(17.3)$ & 0.7503 \\
\hline Other & $2 / 42(4.8)$ & $2 / 23(8.7)$ & 0.5432 \\
\hline PCIS scores (IQR) & $86(80-94)$ & $80(76-90)$ & 0.095 \\
\hline IgG (mg/L) (IQR) & $8.26(5.56-10.3)$ & $10.5(4.965-19.55)$ & 0.4587 \\
\hline IgA (mg/L) (IQR) & $0.82(0.38-1.27)$ & $0.65(0.49-1.87)$ & 0.5965 \\
\hline IgM (mg/L) (IQR) & $0.88(0.62-1.55)$ & $1.02(0.57-1.51)$ & 0.9327 \\
\hline C3 (mg/L) (IQR) & $0.83(0.72-0.98)$ & $0.81(0.63-1.01)$ & 0.9663 \\
\hline $\mathrm{C} 4(\mathrm{mg} / \mathrm{L})(\mathrm{IQR})$ & $0.18(0.14-0.23)$ & $0.19(0.11-0.28)$ & 0.6424 \\
\hline CD3\% (IQR) & $63.06(55.65-74.72)$ & $70.21(61.92-77.22)$ & 0.0716 \\
\hline CD4 (\%) (IQR) & $31.69(23.76-39.83)$ & $35.82(27.32-46.63)$ & 0.2482 \\
\hline CD8 (\%) (IQR) & $23.01(16.93-29.32)$ & $30.23(19.64-37.26)$ & 0.0866 \\
\hline B cell (\%) (IQR) & $26.98(19.83-34.16)$ & $22.26(13.72-26.52)$ & 0.0578 \\
\hline NK cell (\%) (IQR) & $5.03(2.39-8.86)$ & $5.23(3.14-13.17)$ & 0.3278 \\
\hline PICU stay (days) (median, IQR) & $5.00(3.00-7.00)$ & $9.00(3.00-12.00)$ & 0.0124 \\
\hline
\end{tabular}

TABLE 2: Comparison of concentrations of cytokines in serum from nonseptic critically ill children in PICU with or without clinical recovery by day 4 .

\begin{tabular}{lccc}
\hline Cytokines $(\mathrm{pg} / \mathrm{mL})$ & Clinical symptoms relieved at day 4 & No $(n=23)$ & $p$ value \\
\hline IL-2 & $6.39(3.98-9.29)$ & $4.75(3.40-9.34)$ & 0.5356 \\
IL-4 & $3.54(1.66-13.20)$ & $5.31(1.75-15.16)$ & 0.5022 \\
IL-6 & $22.26(5.61-46.19)$ & $40.65(12.66-137.50)$ & 0.0474 \\
IL-10 & $6.04(4.81-20.77)$ & $10.25(6.28-28.08)$ & 0.1369 \\
IL-17A & $16.91(7.73-30.30)$ & $21.31(13.95-42.83)$ & 0.1777 \\
IFN- $\gamma$ & $3.92(3.28-6.08)$ & $4.90(3.56-9.52)$ & 0.1428 \\
TNF- $\alpha$ & $3.25(2.47-4.42)$ & $3.12(2.40-6.42)$ & 0.8496 \\
TNF- $\beta$ & $6.21(4.02-8.19)$ & $4.95(3.85-16.34)$ & 0.8744 \\
G-CSF & $29.47(18.11-49.08)$ & $44.10(19.10-106.00)$ & 0.1321 \\
GM-CSF & $6.32(5.21-10.29)$ & $6.14(3.99-10.62)$ & 0.7234 \\
\hline
\end{tabular}

(55.65\%; 74.72\%)]. The median proportion of $\mathrm{CD}^{+} \mathrm{T}$ cells and NK cells was initially similar to that found in clinically recovered patients.

For the cytokine analysis, IL-2, IL-4, IL-10, IL-17A, IFN- $\gamma$, TNF- $\alpha$, TNF- $\beta$, G-CSF, and GM-CSF levels were not found to be significantly different in patients who clinically recovered compared to patients with disease progression (Table 2). However, patients with disease progression, the level of IL- 6 was significant higher than that in patients who clinically recovered $[40.65 \mathrm{pg} / \mathrm{mL}(12.66 \mathrm{pg} / \mathrm{mL}-137.50 \mathrm{pg} / \mathrm{mL})$ vs. $22.26 \mathrm{pg} / \mathrm{mL}(5.61 \mathrm{pg} / \mathrm{mL}-46.19 \mathrm{pg} / \mathrm{mL} ; p=0.046)]$. The level of CRP in patients with disease progression $[54.37 \mathrm{mg} / \mathrm{L}$ $(18.50 \mathrm{mg} / \mathrm{L}-102.50 \mathrm{mg} / \mathrm{L})]$ was also dramatically higher than that in patients who recovered $[17.64 \mathrm{mg} / \mathrm{L}(3.23 \mathrm{mg} / \mathrm{L}-$ $40.57 \mathrm{mg} / \mathrm{L})](p=0.0083)$ (Figure 1).

3.3. Clinical Recovery by Day 4 Is Associated with Plasma IL-6 Levels in Nonseptic Patients. In the univariate Cox regression analysis, PCIS score and plasma IL-6 levels were associated with outcome at the level of $p<0.1$ (Table 3). Multivariate analysis evidenced that the level of plasma IL- 6 was a factor determining the length of hospital stay (Table 4).

The prognosis value of IL- 6 as a linear variable to predict nonseptic critical illness was calculated with the ROC curve, and the area under the curve was 0.819 (95\% CI: $0.702-0.805 ; p<0.001)$. The optimal threshold value was 


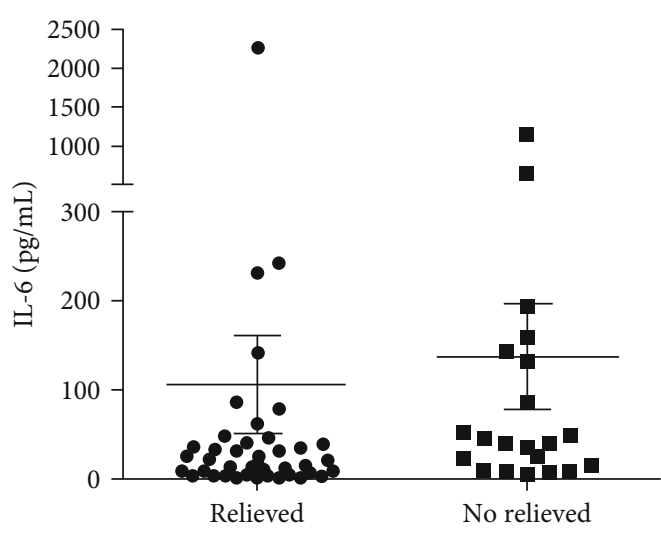

(a)

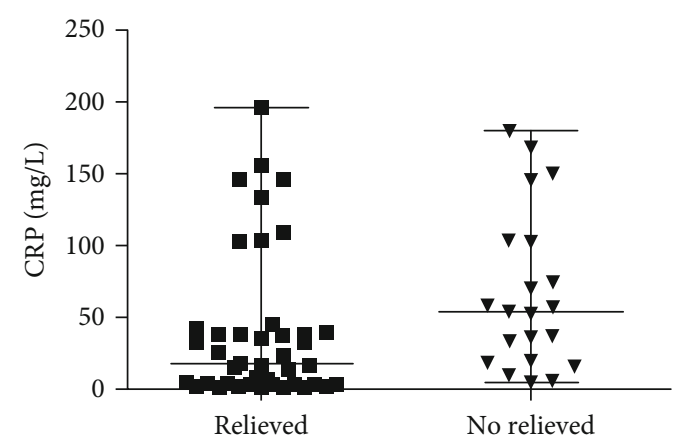

(b)

FIGURE 1: Scatter plots showing IL-6 (a) and CRP (b) concentrations in patients with disease progression versus who clinically recovered.

TABle 3: Multiple linear regression for factors determining PICU length of stay.

\begin{tabular}{|c|c|c|c|c|}
\hline \multirow{2}{*}{ Risk factors } & \multirow{2}{*}{ HR } & \multicolumn{2}{|c|}{ 95\% CI for Exp } & \multirow{2}{*}{$p$ value } \\
\hline & & Lower & Upper & \\
\hline PCIS scores & 0.8022 & 0.8258 & 0.9858 & 0.0880 \\
\hline $\mathrm{CD} 9^{+} \mathrm{B}$ cells & 0.9550 & 0.8913 & 1.0030 & 0.1910 \\
\hline $\mathrm{CD}^{+} \mathrm{T}$ cells & 1.0288 & 0.9696 & 1.0916 & 0.3479 \\
\hline $\mathrm{CD}^{+} \mathrm{T}$ cells & 1.0422 & 0.9774 & 1.1114 & 0.2067 \\
\hline IL-6 & 1.0063 & 1.0017 & 1.0306 & 0.0278 \\
\hline CRP & 1.0078 & 0.9975 & 1.0182 & 0.0139 \\
\hline
\end{tabular}

TABLE 4: Cox regression analysis for prognosis.

\begin{tabular}{lcc}
\hline Risk factors & Coefficient & $p$ value \\
\hline PCIS scores & -0.0812 & 0.1855 \\
CD19 ${ }^{+}$B cells & -0.0033 & 0.9425 \\
CD8 $^{+}$T cells & 0.0533 & 0.2287 \\
$\mathrm{CD}^{+}$T cells & -0.0097 & 0.8439 \\
IL-6 & 0.0265 & $<0.0001$ \\
CRP & 0.0077 & 0.0029 \\
\hline
\end{tabular}

$39.32 \mathrm{pg} / \mathrm{mL}$, when sensitivity was $90.5 \%$ (95\% CI: $69.6-$ $98.8 \%$ ) and specificity was $71.4 \%$ (95\% CI: $55.4-84.3 \%$ ). The total AUC of CRP was 0.706 (95\% CI: $0.578-0.814$; $p=0.0018$ ). The optimal threshold value was $45.85 \mathrm{mg} / \mathrm{L}$, when sensitivity was $57.14 \%$ (95\% CI: $34.0-78.2 \%$ ) and specificity was $90.95 \%$ (95\% CI: 65.9-91.4\%). Otherwise, there was no significant difference between IL- 6 and CRP $(p=0.0934)$ (Figure 2).

\section{Discussion}

The evaluation of immune suppression in critically ill pediatric patients has been previously studied in sepsis [15], but very little data is available on pediatric patients without sepsis. We have shown in this retrospective study that the levels of serum immunoglobulin, serum complement concentrations, lymphocyte subsets, and cytokines (IL-2, IL-4,

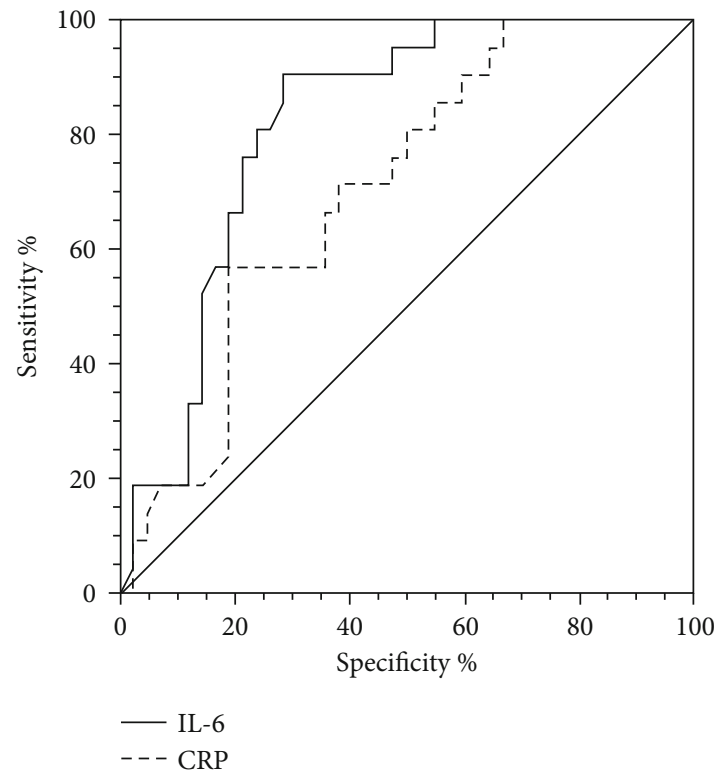

Figure 2: Receiver Operating Characteristic (ROC) curve for IL-6 and CRP (predictor variable) to predict clinically recovered children (outcome variable) among nonseptic critically ill children.

IL-10, IL-17A, IFN- $\gamma$, TNF- $\alpha$, TNF- $\beta$, G-CSF, and GM$\mathrm{CSF}$ ) were not found to be associated with the prognosis of nonseptic pediatric patients, but the plasma IL-6 levels were identified as independently associated with clinical recovery and PICU stay time in nonseptic critically ill children.

Our results demonstrated that early immune monitoring at PICU admission could help to identify those patients with better prognosis. In this setting, we demonstrated that IL-6 levels (with higher $39.32 \mathrm{pg} / \mathrm{ml}$ ) have a high negative predictive value of prognosis in nonseptic critically ill children, which was consistent with the results of previous studies in neonatal/pediatric critically ill patients with sepsis $[16,17]$. CRP was a routinely measured inflammatory marker. The present study showed that both IL-6 and CRP could be considered predictors of the prognosis of nonseptic critically illness. Additionally, the optimal threshold value was higher than that reported by Vasconcellos et al. [18]. They only 
demonstrated that IL-6 levels under $12.5 \mathrm{pg} / \mathrm{mL}$ have a high negative predictive value for pneumococcal infection among these children [18]. Nevertheless, aside from respiratory disease, other systemic diseases including gastrointestinal disease and cardiovascular disease were involved in our study. It could be explained that IL-6 distribution may depend on clinical etiology.

It has been established that IL-6 is a potent pleiotropic cytokine with main proinflammatory effector function, which augments immune responses via induction of $\mathrm{T}$ cell activation, $\mathrm{B}$ cell proliferation, and differentiation, and stimulates acute-phase protein release (e.g., C-reactive protein) [19]. However, our data showed that patients with disease progression had a lower level of B cells, which was not consistent with the trends to that of IL-6. A recent meta-analysis showed that reduced numbers of circulating B cells were negatively associated with sepsis survival [20]. Herein, circulating B cells diminished in both sepsis and nonsepsis patients, which were associated with disease prognosis. This suggested that the relationship between IL- 6 and B cell responses needs to be further explored.

In this cohort of critically ill nonseptic children, there were no significant differences in neither serum immunoglobulin (IgG, IgM, and IgA) nor serum complement (C3, C4) concentrations between children with disease progression and who clinically recovered. The results of this study were not completely consistent with those of other findings in adults. It has been reported in adults that IgM played a protective role in studies because higher levels of this immunoglobulin translated into increased survival [20-22]. Because of the particularity of children in the period of growth and development on the immune system, their immunoglobulins were different from those of adults. It had been reported that immunoglobulins rise to their plateau at age 5 in the toddlers [20-22], whereas the median age of pediatric patients was 3 years in our study; it was suggested that their humoral immune system was immature.

However, this retrospective study has some limitations. First, mHLA-DR presents a potential marker for the identification of immunosuppressed children. Children with decreased mHLA-DR expression developed more secondary acquired infections and more severity sepsis $[1,23]$. The analysis of mHLA-DR on circulating monocytes was not performed. Second, the immune response to critical illness was a dynamic process. Therefore, the time course of different immune parameters should be monitored. Third, previous studies had demonstrated that age and sex could influence variation in immune parameters [24, 25]. Due to the relatively small number of pediatric patients included in this study, we cannot proceed with multivariate analyses potentially associated with immune parameters including age and sex. These limitations need to be further explored, ideally in a multicenter and prospective study.

In conclusion, our results demonstrate that IL-6 levels in the first 72 hours after admission are associated with clinical recovery and PICU stay time in nonseptic critically ill pediatric patients, whereas immunoglobulins and lymphocyte subsets are not. Future studies will need to be conducted to understand the dynamic balance of hyper- and hypoinflam- matory responses in nonsepsis, as well as in sepsis, which could provide valuable insight for making personalized therapeutic schemes targeting immune dysfunction and improving the prognosis of nonsepsis.

\section{Data Availability}

The data used to support the findings of this study are available from the corresponding author upon request.

\section{Conflicts of Interest}

No conflict of interest exits in this manuscript.

\section{Authors' Contributions}

Jianzhong Dang is the first author. Lingfang Tan and Jianzhong Dang contributed equally to this work.

\section{Acknowledgments}

The authors thank the PICU medical team for their dedication to the patients and kindly collecting the samples for the study.

\section{References}

[1] T. F. Manzoli, E. J. Troster, J. F. Ferranti, and M. M. Sales, "Prolonged suppression of monocytic human leukocyte antigen-DR expression correlates with mortality in pediatric septic patients in a pediatric tertiary intensive care unit," Journal of Critical Care, vol. 33, pp. 84-89, 2016.

[2] R. A. Devine, Z. Diltz, M. W. Hall, and R. K. Thakkar, "The systemic immune response to pediatric thermal injury," International Journal of Burns and Trauma, vol. 8, no. 1, pp. 6-16, 2018.

[3] E. Sarmiento, J. Navarro, J. Fernandez-Yanez, J. Palomo, P. Munoz, and J. Carbone, "Evaluation of an immunological score to assess the risk of severe infection in heart recipients," Transplant Infectious Disease, vol. 16, no. 5, pp. 802-812, 2014.

[4] S. Iwase, T. A. Nakada, N. Hattori et al., "Interleukin-6 as a diagnostic marker for infection in critically ill patients: a systematic review and meta-analysis," American Journal of Emergency Medicine, vol. 37, no. 2, pp. 260-265, 2019.

[5] A. A. Yousef, Y. M. Amr, and G. A. Suliman, "The diagnostic value of serum leptin monitoring and its correlation with tumor necrosis factor- $\alpha$ in critically ill patients: a prospective observational study," Critical Care, vol. 14, no. 2, p. R33, 2010.

[6] M. L. Berres, B. Schnyder, E. Yagmur et al., "Longitudinal monocyte human leukocyte antigen-DR expression is a prognostic marker in critically ill patients with decompensated liver cirrhosis," Liver International, vol. 29, no. 4, pp. 536-543, 2009.

[7] S. Harbarth, K. Holeckova, C. Froidevaux et al., "Diagnostic value of procalcitonin, interleukin-6, and interleukin- 8 in critically ill patients admitted with suspected sepsis," American Journal of Respiratory and Critical Care Medicine, vol. 164, no. 3, pp. 396-402, 2001.

[8] C. Cheval, J. F. Timsit, M. Garrouste-Orgeas et al., "Procalcitonin (PCT) is useful in predicting the bacterial origin of an 
acute circulatory failure in critically ill patients," Intensive Care Medicine, vol. 26, no. S2, pp. S153-S158, 2000.

[9] L. Weidhase, D. Wellhofer, G. Schulze et al., "Is interleukin-6 a better predictor of successful antibiotic therapy than procalcitonin and C-reactive protein? A single center study in critically ill adults," BMC Infectious Diseases, vol. 19, no. 1, p. 150, 2019.

[10] M. L. Washburn, Z. Wang, A. H. Walton et al., "T cell- and monocyte-specific RNA-sequencing analysis in septic and nonseptic critically ill patients and in patients with cancer," Journal of Immunology, vol. 203, no. 7, pp. 1897-1908, 2019.

[11] M.-. S. L. Y. Koning, W. A. C. (. K. Koekkoek, J. C. N. (. H.). Kars, and A. R. H. van Zanten, "Association of protein and caloric intake and clinical outcomes in adult septic and nonseptic ICU patients on prolonged mechanical ventilation: the PROCASEPT Retrospective Study," Journal of Parenteral and Enteral Nutrition, vol. 44, no. 3, pp. 434-443, 2020.

[12] J. J. Li, Y. F. Chen, and Y. X. Lin, "Investigation of disease spectrum in the PICU of Shengjing Hospital of China Medical University between 2005 and 2012," Zhongguo Dang Dai Er Ke Za Zhi, vol. 15, no. 6, pp. 472-476, 2013.

[13] H. X. Dang, C. J. Liu, J. Li, S. J. Chen, and F. Xu, "Clinical significance and prognostic effect of serum 25-hydroxyvitamin D concentrations in critical and severe hand, foot and mouth disease," Nutrients, vol. 9, no. 5, p. 478, 2017.

[14] A. J. Hessels, J. Liu, B. Cohen, J. Shang, and E. L. Larson, "Severity of illness measures for pediatric inpatients," Journal for Healthcare Quality, vol. 40, no. 5, pp. e77-e89, 2018.

[15] J. A. Muszynski, R. Nofziger, M. Moore-Clingenpeel et al., "Early immune function and duration of organ dysfunction in critically III children with sepsis," American Journal of Respiratory and Critical Care Medicine, vol. 198, no. 3, pp. 361-369, 2018.

[16] L. Shahkar, A. Keshtkar, A. Mirfazeli, A. Ahani, and G. Roshandel, "The role of IL-6 for predicting neonatal sepsis: a systematic review and meta-analysis," Iranian Journal of Pediatrics, vol. 21, no. 4, pp. 411-417, 2011.

[17] S. K. Angurana, A. Bansal, S. Singhi et al., "Evaluation of effect of probiotics on cytokine levels in critically ill children with severe sepsis: a double-blind, placebo-controlled trial," Critical Care Medicine, vol. 46, no. 10, pp. 1656-1664, 2018.

[18] A. G. Vasconcellos, J. Clarencio, D. Andrade, M. A. Cardoso, A. Barral, and C. M. Nascimento-Carvalho, "Systemic cytokines and chemokines on admission of children hospitalized with community-acquired pneumonia," Cytokine, vol. 107, pp. 1-8, 2018.

[19] C. A. Pfortmueller, C. Meisel, M. Fux, and J. C. Schefold, "Assessment of immune organ dysfunction in critical illness: utility of innate immune response markers," Intensive Care Medicine Experimental, vol. 5, no. 1, p. 49, 2017.

[20] C. Krautz, S. L. Maier, M. Brunner et al., "Reduced circulating B cells and plasma IgM levels are associated with decreased survival in sepsis - a meta-analysis," Journal of Critical Care, vol. 45, pp. 71-75, 2018.

[21] D. Andaluz-Ojeda, V. Iglesias, F. Bobillo et al., "Early levels in blood of immunoglobulin $\mathrm{M}$ and natural killer cells predict outcome in nonseptic critically ill patients," Journal of Critical Care, vol. 28, no. 6, pp. 1110.e7-1110.e10, 2013.

[22] M. Justel, L. Socias, R. Almansa et al., "IgM levels in plasma predict outcome in severe pandemic influenza," Journal of Clinical Virology, vol. 58, no. 3, pp. 564-567, 2013.
[23] S. Remy, K. Kolev-Descamps, M. Gossez et al., "Occurrence of marked sepsis-induced immunosuppression in pediatric septic shock: a pilot study," Annals of Intensive Care, vol. 8, no. 1, p. 36, 2018.

[24] L. Jia, J. Li, Y. Zhang et al., "Age- and sex-related reference intervals of lymphocyte subsets in healthy ethnic Han Chinese children," Cytometry Part A, vol. 87, no. 12, pp. 1116-1126, 2015.

[25] W. T. Shearer, H. M. Rosenblatt, R. S. Gelman et al., "Lymphocyte subsets in healthy children from birth through 18 years of age: the pediatric AIDS Clinical Trials Group P1009 study," The Journal of Allergy and Clinical Immunology, vol. 112, no. 5, pp. 973-980, 2003. 